\title{
Foci of Giant Cell Lesion in the wall of a Radicular Cyst- Case Report
}

\section{Bhaumik Debamalya, BDS ${ }^{1}$, Banerjee Abhishek, $\mathrm{MDS}^{2^{*}}$, Saha Alangkar, MDS $^{3}$, Pramod J, MDS ${ }^{4}$, Banerjee Sumantro, BDS ${ }^{1}$}

${ }^{1}$ Post Graduate Trainee, Oral and Maxillofacial Pathology, Awadh Dental College Hospital, Jamshedpur

${ }^{2}$ Assistant Professor, Oral and Maxillofacial Pathology, Awadh Dental College Hospital, Jamshedpur

${ }^{3}$ Clinical Tutor cum Demonstrator, Oral and Maxillofacial Surgery, Bardhaman Dental College Hospital, Burdwan, WB

${ }^{4}$ Professor and Head, Oral and Maxillofacial Pathology, Awadh Dental College Hospital, Jamshedpur *Corresponding Author

Dr Abhishek Banerjee

Assistant Professor, Oral and Maxillofacial Pathology, Awadh Dental College Hospital, Jamshedpur, India

Abstract
A rare case of unilocular radiolucency associated with the apices of the left maxillary central and lateral
incisor and canine in a 32 years old female is presented. Histological, the lesion showed foci of giant cell
lesion in the wall of radicular cyst, there was no evidence of hyper parathyroidism. The clinic-pathological
presentation is likely a histo-pathological curiosity and may be an example of "double" pathology/collision
of apical inflammatory pathology and central giant cell lesion or reflect florid osteoclastic resorption.
Keyword: Unilocular radiolucency, hyper parathyroidism, "double" pathology.

\section{Introduction}

The large mononucleated or multinucleated giant cells are seen in a variety of physiological as well as pathological conditions. Multinucleated giant cells (MGCs) are important mediators of tissue remodeling and repair and also for removal of foreign materials and various pathogens. The giant cell lesions of oral cavity have been classified on the basis of etio-pathogenesis. The basic information regarding the mechanism of formation and morphology of giant cells and its significance is the associated giant cell lesions. ${ }^{[1]}$ Foreign body giant cells, Langhan's giant cells, Touton giant cells, tumor giant cells, are various types of giant cells, apart from this miscellaneous types of giant cells such as Aschoff,s giant cells of rheumatic nodule and Reed Sternberg cells of Hodgkin's lymphoma etc are the pathologically presented giant cells. ${ }^{[1]}$

It is a large mass forming, by the union of several distinct cells. They are usually very large cell found of by mononuclear cell or by nuclear division without cytoplasmic division of monocytes or macrophage lineage. Osteoclasts in the bones, trophoblasts in placenta, megakaryocytes in the bone marrow, etc. are the physiologically present multinucleated giant cells. [2]

The giant cells may be mononucleated or multinucleated which can be explained by the mechanism of their formation. They are easily recognized under light microscopy. However it is 
essential to know the histogenesis of these unique cells, as the presence of giant cell can have a major implication on the disease process. ${ }^{[2]}$

The giant cells showing variations morphology and functional patterns in various oral lesions. ${ }^{[2]}$ It were first reported in tuberculosis granulomas by Rokitansky and Langhans over a century ago. These giant cells are now recognized as a common feature of granulomas induced both by immunological \& non-immunological stimuli. Thus, they are found in granulomas associated with the immune response to tuberculosis, leprosy, syphilis, \& various fungal \& parasitic infections as well as granulomas associated with non-immune responses to toxic agents such as silica, beryllium, \& asbestos, \& to nontoxic agents such as carbon particles, plastic beads, \& iron particles. $^{[3]}$

The origin $\&$ the mechanism of the formation of multinucleated giant cells are not clear. It is possible, because of the variety of agents that produce granulomas, that these cells are formed by several different mechanism. ${ }^{[3]}$

But two theories were considered about the formation of giant cell, they are:

1. Amitotic division of monocyte nuclei cellular division.

2. Fusion of non-replicating monocytes. ${ }^{[2]}$

Osteoclasts and foreign body giant cells have revealed a number of common factors, e.g., vitronectin, an adhesion protein, dendritic cellspecific transmembrane protein (DC-STAMP), a fusion factor, and macrophage fusion receptor (MFR), that contribute to giant cell formation and function. Insight into common molecules, receptors, and mediators of the adhesion and fusion mechanisms of giant cell formation have been complicated by the wide diversity of species, models, and cell types utilized in these studies. ${ }^{[4]}$

The well-known osteoclast receptor, $\alpha v \beta 3$, may serve as potential therapeutic targets for the modulation and inhibition of multinucleated giant cell formation and function. The intracellular and intercellular signaling mechanisms modulating multinucleated giant cell formation and function are necessary for the identification of therapeutic targets as well as a better understanding of giant cell biology. ${ }^{[4]}$

Multinucleated giant cells are one of the characteristic features of granulomas and are able to attack extracellular material, such as larger pathogens and foreign material. Thus, these cells are able to sequester irremovable material or persistent pathogens to prevent further spread of infection and isolate foreign particles. ${ }^{[5]}$

\section{Case Report}

A 32 years old female was examined in January 2018 for pain in the area of the left maxillary anterior teeth. The medical history was unremarkable. On clinical examination, the left maxillary lateral incisor and canine were sensitive upon percussion. The submitted orthopantomograph showed a circumscribed, unilocular radiolucency surrounding the apices of these teeth (Figure 1); the canine had been subjected to root canal treatment. The lateral incisor was similarly treated and this was followed by surgical exploration of the radiolucency, removal of contents and curetting of the body cavity, apicoectomy of both teeth, and retrograde apical sealing of the canine. There were no postoperative complications. The removed specimen consisted of an opened soft sac, measured 20x8 mm; were fixed in $10 \%$ formalin and routinely processed for histological examination. The sections showed concave, variably and subacutely inflamed, fibrous connective tissue that included two illdefined cellular areas. Epithelial lining was sparse and in bits (Figure 2). The cellular areas showed the typical histology of central giant cell lesion. There are evidence of giant cells in large numbers showing multinucleation without significant arrangements (Figure 3, 4). Levels of calcium, phosphorus, parathyroid hormone and alkaline phosphatase in serum, were within normal limits. 


\section{JMSCR Vol||07||Issue||05||Page 493-499||May}

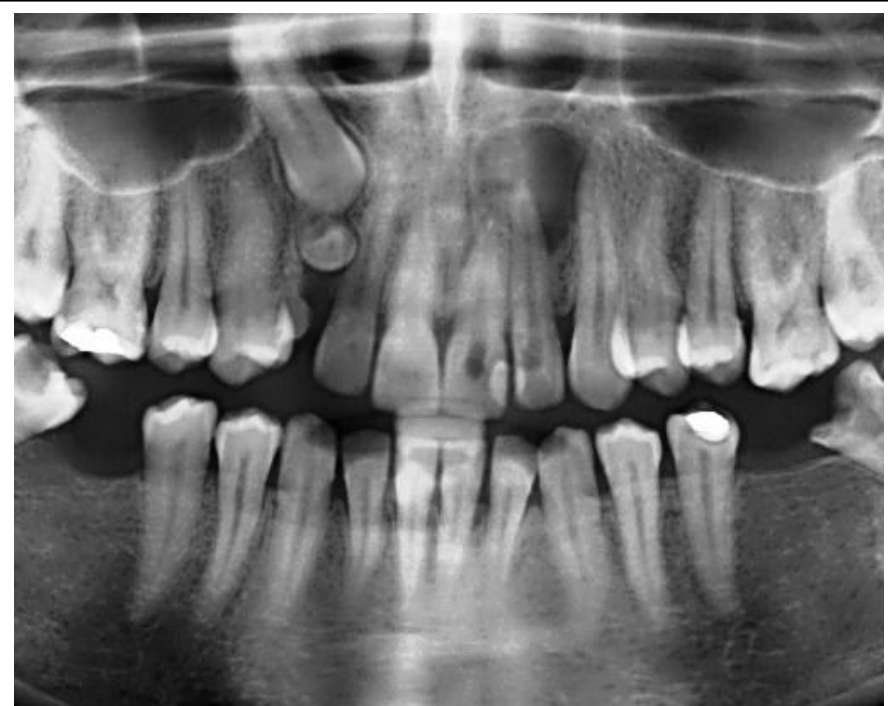

Figure 1 Radiograph showing unilocular radiolucency

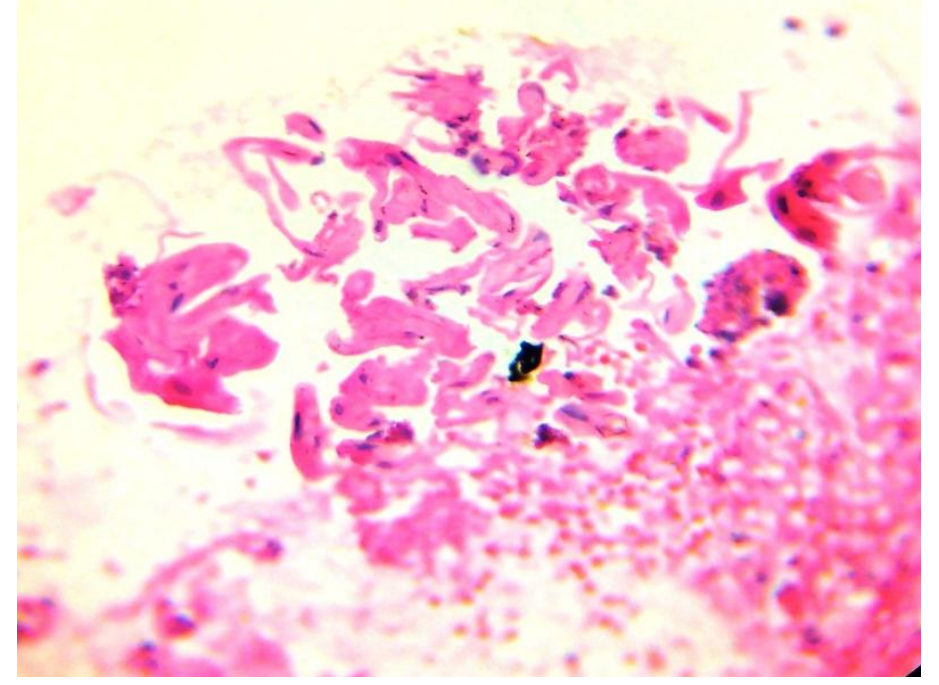

Figure 2 Friable Epithelial bits in radicular cyst

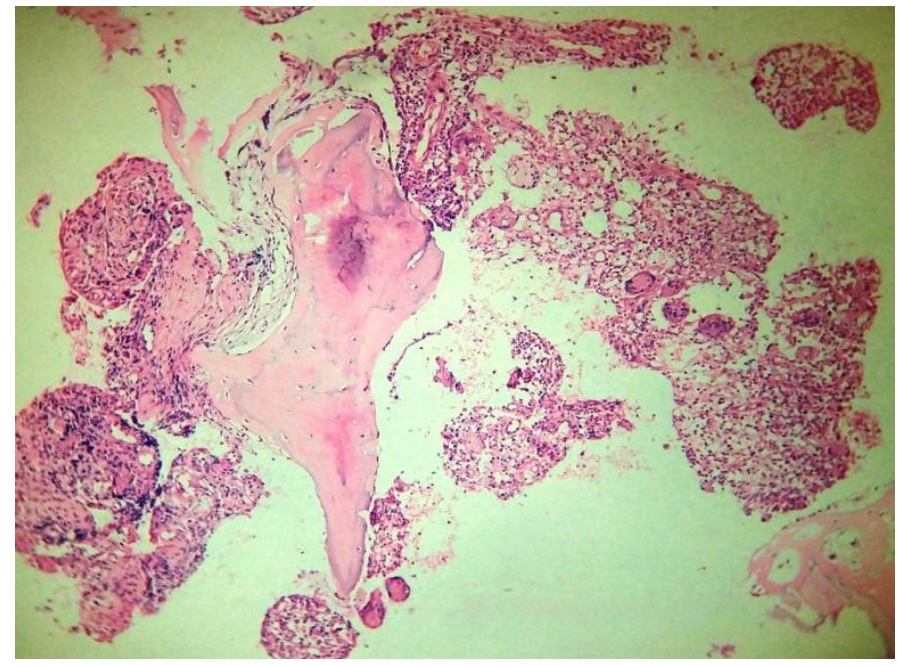

Figure 3 Foci of budding giant cell lesion

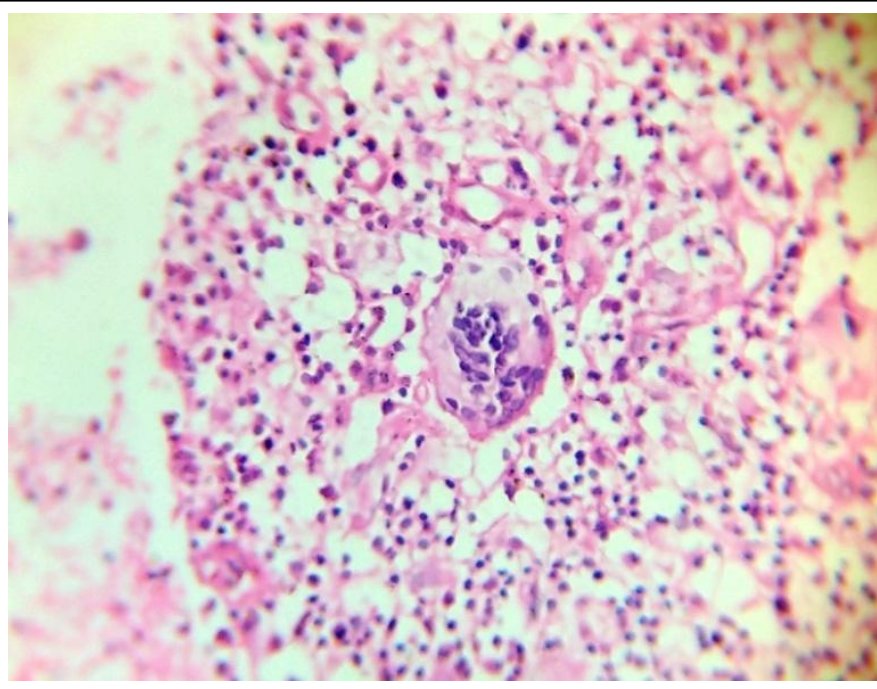

Figure 4 Giant cell, multinucleated without definite pattern of nuclear arrangement.

\section{Discussion}

Inflammatory jaw cysts is a group of odontogenic lesions. They originate as epithelial residues in the periodontal ligament due to apical periodontitis following the death and necrosis of the dental pulp. Radicular cysts are diagnosed either during routine radiographic examination or during the clinical examination after following discoloring of the tooth.

Occurance of the radicular cysts in the maxilla is $60 \%$ as compared with mandible, and it is associated with buccal/labile or palatal enlargement. The present case was associated with a mild labile swelling near the base of the nose, without any palatal involvement.

Radicular cysts grow slowly and lead to mild mobility, root resorption and displacement of teeth and once cyst become infected, may lead to pain and swelling near the base of the nose and erosion and fluctuation of the overlying soft tissue. The bone in the surrounding area will be thinned out with springiness and egg shell crackling, leading to cortical plate expansion. The alveolar process exhibits a paper-like texture on palpation. ${ }^{6}$

A giant cell is a cell, that is larger in dimension than the other cells and these cells are involved in many physiologic and pathological processes. This may be mono-nucleated or multinucleated and are important mediators for tissue remodeling and repair and also help for removal of foreign 
materials. Osteoclasts in the bones, megakaryocytes in the bone marrow, etc. are the physiologically present multinucleated giant cells. $^{7}$

Very few cases are seen in the first decade, after which there is a fairly steep rise, with a peck frequency in the third decade. There are large number of cases in the fourth and fifth decade, after which there there is a gradual decline. A very similar age distribution was reported by Donath (1985).The low frequency in the first decade has been shows in a number of studies and indicateds that although dental caries is very common in children, radicular cysts are not always found associated with deciduous teeth.

Radicular cyst were most commonly seen in men than the female. $58.5 \%$ were seen in male and $41.5 \%$ were in women. The lower frequency in women, may be because they are likely neglect their teeth, particularly the maxillary anterior incisors, where most radicular cyst occur.

They occur in all tooth bearing area of the jaws, although about $60 \%$ are found in the maxilla and $40 \%$ in the mandible The most frequently in the maxillary anterior region, there are a number of possible reason for this is to the hazard by dental caries, maxillary incisors are consequent high risk to the their pulps, Seconds, there is the high palatal invaginations in the maxillary lateral incisors and there frequency of pulp death. Third, maxillary anterior teeth are probably more prone tramatic injuries which lead to pulp death.

Many radicular cysts are symptom less and are discovered when periapical radiographs are taken of teeth with non-vital pulp. In the maxilla there may be buccal or palatal enlargement, whereas in the mandible it is usually labial or buccal and rarely lingual.

Pain and infection are the clinical feature of some radicular cyst. It is painless unless infected.

Residual radicular cysts are retained after removal of non vital tooth. Acute and chronic inflammatory cell infiltration shows variable intensity and their relationship between the percentage of polymorphonucuclear leucocytes in the inflammatory infiltrate and cortication of the cyst wall radiographically.

The mean diameter of the cysts was $1.94 \mathrm{~cm},{ }^{8}$ Histologically, the lesion fulfilled the criteria for radicular cysts ${ }^{8}$. They consisted of a cystic wall lined by stratified non-keratinized squamouus epithelium, sometimes arranged in interconnecting rings, which exhibited spongiosis and exocytosis and was hyperplastic or atrophic. Chronic inflammatory infiltrate, multinucleated giant cells and neutrophils were observed in the cysts ${ }^{8,9}$.

Ciliated eoithelia is uncommon in radicular cyst $^{9,10}$, pseudostratified ciliated epithelium was exclusively observed in RC's located in the anterior region of the maxilla, were identified by Takeda et.al ${ }^{8}$ and Nair et.al ${ }^{9}$.

Apoptosis is a mechanism of genetically programmed cell death which plays an important role in the control of cell population ${ }^{10}$. In almost $30 \%$ of apoptosis cells were detected by Loyola et al. these cells exhibiting mainly atrophic epithelium by using bcl-2 immunostaining. However, Suzuki et al, ${ }^{10}$ using $\mathrm{p}^{53}$, Bax,caspase-3, Fas and Fas-L, concluded that both proliferative activity and apoptosis are more frequent in RC's exhibiting a thick epithelium. This finding is in agreement with the present result as apoptotic cells were seen in most lesion containing a hyperplastic epithelium.

Rushton bodies or hyaline bodies producing a wide variety of shape, including liner, round lamellar or amorphous structure were found in the epithelium of $\mathrm{RCs}^{12,13}$

The presence of these structures depends on the sectioning plane of the material, with their incidence ranging from $2.6 \%$ to $10.3 \%$. In the present study, Rushton bodies were detected in few RCS

The presence of these structures depends on the sectioning plane of the material, with their incidence ranging from $2.6 \%$ to $10.3 \%$. In the present study, Rushton bodies were detected in few RCS

The presence of these structure depends on the sectioning plane of the material, with their 
incidence ranging from $2.6 \%$ to $10.3 \%$.Rushton bodies were dected in few RCs.

Russell bodies also were formed by the accumulation of immunoglobulins in endoplasmic reticulum found in chronically inflamed tissue, periapical lesion rich in Russell bodies and plasma cells. Macrophages are involved in the innate response of the periapical lesion, phagocytosing bacteria and preventing the dissemination of infection, as well as in the acquired response, presenting antigens to lymphocytes through the MHC II system ${ }^{14}$.

Exogenous material was observed close to the mineralization foci and foreign body -type reactions. Foreign body reactions occur concomitantly with intense inflammatory infiltration containing macrophages and multinucleated giant cell. ${ }^{15}$

The pathogenesis of Giant Cell is poorly understood. The epidemiology of Giant Cell strongly indicates that genetic background, ageing and gender undoubtedly play a role. Various polymorphisms have been associated with increased risk of Giant Cell, but the strongest association appears to be with variants in the class II major histocompatibility complex reinforcing the concept that GC is an antigen-driven disease. Activated dendritic cells are present in lesions and are thought to play an important role in T-cell activation. Th1-mediated immune response, with vigorous expression of IFN $\gamma$ and IFN $\gamma$ - induced products in lesions in accordance the granulomatous nature of lesions. A Th17mediated immune response also contributes to GC and that patient with prominent Th17 response respond better to glucocorticoid treatment.

IFN $\gamma$ is a potent activator of macrophages which maintain inflammatory cascades and participate in vascular injury. Macrophages produce proinflammatory cytokines IL-1, TNF $\alpha$ and IL-6 among many others which correlate with the intensity of the systemic inflammatory response, typical of the disease. Tissue expression and serum concentrations of TNFa and IL-6 correlate with disease persistence. Chemokines, endothelial adhesion molecules and colony-stimulating factors are also produced in lesions and reinforce inflammatory loops by recruiting and expanding the half-life of additional inflammatory cells.

Matrix metalloprotease (MMP-9 and MMP-2) expression, activation and proteolytic activity have been detected in lesions and, given their elastinolytic activity, are probably contributing to disruption of elastic fibres and abnormal vascular remodelling

The association between increased expression of $\mathrm{TNF} \alpha$ and persistent disease activity provided support to the performance of clinical trials blocking TNF and blocking the IL-6 receptor with tocilizumab is being tested in an international multicentre trial. IL-6 is a multifunctional cytokine involved not only in inducing the acute phase response and ensuing systemic symptoms but also in maintaining the Th17 pathway. Interfering with CD28-mediated T-cell costimulation with abatacept, presumably during antigen presentation, is also currently being tested in a multicentre trial.

Growth factors produced remodelling programme leading to myofibroblast differentiation of smooth muscle cells, migration towards the intimal layer and deposition of extra cellular matrix proteins. This leads to intimal hyperplasia and vessel occlusion, with. Several factors including PDGFs, TGF $\beta$ and endothelin-1,

\section{Immunohistochemistry}

Samples of periapical cyst obtained from 12 patients were examined using light and electron microscopes and monoclonal antibodies. Monocytes/macrophages, lymphocytes, and plasma cells were nearly always the most huge cell populations. Ultrastructural analysis showed close contacts between macrophages and cells of the lymphoid lineage, with the lymphoid cells frequently demonstrating blastic features.

Immunohistochemical staining with the antiinterleukin 2 receptor antibody showed that the concentration of labeled cells was quite low. The vast majority were lymphocytes, though some mast cells were also labeled. Mast cells were 
chiefly located in perivascular areas and interleukin 2 receptor-positive mast cells were frequently associated with lymphoid cells, mast cells could be part of a negative feedback mechanism in the immune response. By releasing histamine, they would block the immune response and by absorbing interleukin 2 they would remove it as an immune system stimulant.

Radicular cysts contain cavities which completely enclosed by epithelial lining, and present remitted inflammatory response than that in granu- loma. Studies have shown periapical lesions infiltrated by different inflammatory cells such as lymphocytes.

Multiple subpopulations of immune cells are found in periapical lesions, while CD8 + and CD4 $+\mathrm{T}$ cells seem to be the dominant lymphocytes in periapical granuloma. Appropriate activation of antigen-specific $\mathrm{T}$ cells leads to their proliferation and acquisition of effector function

Radicular cysts were diagnosed as below:

(a) a lesion situated on the periapical region of a non-vital tooth.

(b) a cavity with fluid or semisolid content.

(c) histologic observation of stratified nonkeratinizing squamous epithelium lining a cystic cavity. Dental granulomas were diagnosed by the observation of a chronic inflammatory reaction, with a large number of infiltrating inflammatory cells (macrophages, lymphocytes and plasma cells) . The control group was healthy oral mucosa tissues $(n=6)$, they were obtained from residual mucosa after third molar extraction which did not show any inflammation.

\section{Histopathological Features}

The gross specimen may be ovoid cystic masses, but they are irregular $\&$ collapsed. The walls vary from extremely thin to a thickness of about $5 \mathrm{~mm}$. The inner surface may be smooth or corrugated. The histopathological studies shows following features---

1) Epithelial Lining: All radicular cysts are lined by Stratified Squamous Epithelium and 1 to 50 cell layers thick. In such cases of cyst is lined with a pseudo stratified cilliated columnar epithelium or respiratory type of epithelium. Ortho or parakeratinised linings are very rarely seen in radicular cysts. Secretary cells or ciliated cells are frequently found in epithelial lining

2) Rushton's Hyaline Bodies: $10 \%$ cases of radicular cysts, Rushton's Hyaline bodies are found in epithelial linings. Very rarely they are found in Fibrous capsule. The hyaline bodies are tiny linear and shaped bodies which are amorphous in structure, eosinophillic in reaction and Brittle in nature.

3) Cholesterol Clefts: Cholesterol crystals are found in many radicular cysts, slow considerable amount of cholesterol accumulation could disintegation of lymphocytes, plasma cells \& macrophages taking part in inflammatory process, with consequent release of Cholesterol release from their walls.

4) Fibrous Capsule: In Radicular Cyst the Fibrous Capsuleis composed of mainly condensed parallel bundles of collagen fibres peripherally and a loose connective tissue adjacent to epithelial lining.

5) Inflammatory Cells: During epithelial proliferation acute inflammatory cells are present.In the connective tissue immediately adjacent to the epithelium,chronic inflammatory cells are present.

6) Remnant of Odontogenic Epitheliam, Mast cells and sometime Stallite microcysts are also present. Some cystare highly vascularised and different limit of calcification are also present.

\section{References}

1. Varghese I, PrakashA :Giant cell lesions of oral cavity : Oral \& Maxillofacial Pathology 2011; ISSN 0976- 1225

2. Shrestha A, Marla V, Shrestha S, Neupane M. Giant cells and giant cell lesions of oral cavity - a review: CDJ 2014; 17(2); 192204

3. https/medical-dictionary. thefreedictionary.com/giant+cell. 
4. Branehog I, Ridell B, Swolin B, Weinfeld A (1975). "Megakaryocyte quantifications in relation to thrombokinetics in primary thrombocythaemia and allied diseases". Scand. J. Haematol. 15 (5): 32132. doi:10.1111/j.16000609.1975.tb01087.x. PMID 1060175.

5. Krishnamurthy V, Haridas S, Garud M, et al. Radicular cyst masquerading as a multilocular radiolucency. Quintessence Int 2013;44:71-3 [PubMed]

6. Joshi N, Sujan SG, Rachappa MM. An unusual case report of bilateral mandibular radicular cyst. Contemp Clin Dent 2011;2:59-62 [PMC free article] [PubMed]

7. Schramm A, Rucker M, Sakkas N, et al. The use of cone beam CT in cranio maxilla facial surgery. Int Congress Series 2005;1281:1200-4

8. Simon JHS. Incidence of periapical cysts in relation to the root canal. J Endod 1980;6:845-8 [PubMed]

9. Nair PNR. Non-microbial etiology: periapical cysts sustain post-treatment apical periodontitis. Endod Top 2003; 6:96-113

10. Jacob S. Rushton or hayline bodies in radicular cysts.A morphologic curiosity. Indian J Pathol Microbiol 2010;53:846-7 [PubMed]

11. Swinson BD, Jerjes W, Thomas GJ. Squamous cell carcinoma arising in a residual odontogenic cyst: case report. J Oral Maxillofac Surg 2005;63:1231-3 [PubMed]

12. Tandri SB. Management of infected radicular cyst by surgical decompression. $\mathrm{J}$ Cons Dent 2010;13:159-61 [PMC free article] [PubMed]

13. Lagares DT, Segura-Egea JJ, Caballero AR. et al. Treatment of large maxillary cyst with marsupilization, decompression, surgical endodontic therapy and enucleation. J Can Dent Assoc 2011; 77:b87. [PubMed]

14. Dominiak M, Lysiak-Drwal K, Gedrange $\mathrm{T}$, et al. Efficacy of healing process of bone defects after apectomy: results after 6 \& 12 months. J Physiol Pharmacol 2009;60(Suppl 8):51-5 [PubMed]

15. Yoshikawa G, Murashima Y, Wadachi R, et al. Guided bone regeneration using membranes and calcium sulphate after apicectomy: a comparative histomorphometrical study. Int Endod J2002;35:25563 [PubMed]. 